\title{
The Influence of User Network Environment on Reward Sharing Behavior in Social Media
}

\author{
Guo Bing ${ }^{1, a,{ }^{*}}$ \\ ${ }^{1}$ Nanjing University of Science and Technology, Jiangsu, China \\ aguobing430722@163.com \\ *132-3657-0096
}

Keywords: Social media, Social network, Reward sharing behavior.

\begin{abstract}
For the study of user sharing behavior in social media, the network environment and incentive mechanisms are two important aspects. Based on the social network theory, this paper put forward the hypothesis, using crawler technology to grab user data on Sina Microblog platform. Based on 1774 samples, using SPSS software to carry out descriptive statistics, correlation analysis and regression analysis. The research shows that the network density in social media positively influences the user's reward-sharing behavior, network site has no significant effect on the reward sharing behavior of users.
\end{abstract}

\section{Introduction}

In the era of mobile Internet, the application and research of social media has always been the focus of attention from all walks of life. Emerging industries such as we-media, live broadcast and short video which are effective channels for internet celebrity and brand owners to expand their influence. The effect of brand information recommendation by users in social media is better than traditional offline promotion [1]. Brand owners use it to find target customers, communicate with customers, publish advertisements, and provide brand services [2]. But users are the key to the development of social media [3]. The research on individual users has attracted attention of all circles, accurate marketing and personalized recommendation have become common ways in marketing. So what are the factors influencing users' reward sharing behavior in social media and how are they measured? Can brand owners achieve efficient and widespread dissemination through customized marketing strategies? This is the focus of this article.

\section{Literature review and hypothesis development}

\subsection{Reward sharing behavior}

The information sharing behavior is divided into spontaneous sharing and incentive sharing. Rewardsharing behavior belongs to the latter, it gives a certain spiritual or material reward to the user for his or her information sharing behavior, which is the common ways to attract the attention of the consumer by the brand. Erdelez and Rioux believe that user share information is due to interest, altruism, sharing is convenient and competing with others [4]. Lin argue that the user's information sharing behavior can gain internal and external benefits, such as self-disclosure, honorary benefits, reciprocal relationships and external remunerations [5]. In addition, Sun's study on commentary communities show that, when user have weak relationship, incentive mechanisms can increase the number of reviews by 14 times, while those with strong relationships are reduced by $90 \%$ [3]. Hui and Liaogang study the impact of the incentive mechanism on reviews of Taobao, which can improve the reputation of online stores, but the effect is affected by the consumer's satisfaction towards the product [6]. Consumer's purchase intention is influenced by the word of mouth, and study find that the effect of word-of-mouth produced by incentive mechanism is less than the natural word-of-mouth [6]. 
Reward sharing behavior is popular on Sina Microblog platform, which has different requirements for users (i.e., @ a certain number of friends), and the winners select from the eligible users. Launching reward-sharing activities through the lottery platform can attract more users to follow the account. Since incentive effect is affected by the level of effort that the user needs to make [6], it is not suitable to set high threshold.

\subsection{Social network}

Early research mainly focused on the social network structure itself and its impact on residents' social behavior, it is widely used to study user network behavior nowadays. Social networks are divided into two types: ego-centric networks and whole networks, the former is a network structure formed around a specific node, and the latter is a network of all nodes [7]. This paper uses individual centers network. Network size describes the number of nodes in the formed network [7,8], it refers to the total number of network members in social media $[9,10]$. Network density describes the closeness of the connection between nodes, it means the ratio of the actual number of connections to the maximum number of possible connections in the network [8], connection refers to interaction in social media $[3,9]$. Early scholars had used social network theory to study the channels, characteristics, audiences, and influencing factors of information sharing in the online community [10], and also studied the differences in the user's relationship and the network response behavior under the incentives [3]. However, there are arguments about the research results. For example, Kivran Swaine and Naaman, Lingling and Mi believe a large and lean network structure that helps users spread information [9], while Hongtao point out that a close network can help users share information [8].

A large network size means user has more friends or fans in social media, and there are more readers after the content is published, which can bring interaction [10]. Meanwhile, friends and fans mean that users already possess social capital [7]. Through the reward sharing behavior, one can indirectly show his concerns [9], stereotype network personas settings, and obtain reputation gains [5]. Otherwise, it can recommend excellent bloggers or content to readers [4], demonstrate a willing to share and enhances popularity [5]. Research show that large-scale audiences would stimulate users to contribute public content in order to obtain potential social benefits [11]. Lin and Qiu found that users with a large number of fans are more willing to disclosure their emotions [10]. Thus, we hypothesize:

H1: network size has a positive impact on the user's reward sharing behavior.

There is a strong correlation between user content publishing and the network environment [3]. High network density means frequent interaction and close interpersonal relationships, which can promote resource sharing [11]. Park et al. studied the Facebook use behavior of college students, found that the proximity and density of individual networks had positive correlation with information sharing [11]. Microblog is a kind of information, users are more likely to be stimulated by the incentive mechanism in a strong-tie environment [6], and more likely to share the reward-sharing information. On the one hand, it is driven by economic benefits (prizes). on the other hand, a certain internal income can be obtained if no external remuneration is obtained, such as reputation benefits (i.e., insight, taste, and humor) [5]. At the same time, the risk is small because high density network structure is accompanied by higher solidarity, support and trust [10]. If face risk arises, users can choose to delete micro-blog afterwards. So, we hypothesize:

H2: network density has a positive impact on user's reward sharing behavior.

\section{Empirical analysis}

\subsection{Data collection and processing}

This paper takes Sina Micro-blog as research platform, and uses the official micro-blog of Smartisan phone as research object, using the Python to compile the web crawler program and collect original data. March to April 2018, 6231 initial user ID were obtained. Since this article mainly study the reward sharing behavior of individual users in social networks. Network density calculation requires a network size not less than 1 and to avoid user use micro-blog do marketing things, limit the number 
of fans 1 1000. In order to exclude users from publishing large amounts of useless information and spam, limit the number of micro-blogs to $1 \sim 2000$. After cleaning the invalid data according to the above conditions, 1774 valid sample users and 892,647 micro-blog data were obtained.

\subsection{Variable measurement}

Network size refers to the effective scale of browsing [11], fans can only receive bloggers' pushing and the blogger can't receive fans' pushing in Sina micro-blog, so network size is equal to the number of fans. Network density refers to the ratio of all generated connections to all possible connections with fans [7], connection refers to the sum of sharing, comment and like.

\subsection{Descriptive analysis}

In the sample, men accounted for $69.17 \%$ and women for $30.83 \%$, and $68.15 \%$ of the users are $18 \sim 24$ years old. Users in East, Central, and South China respectively accounted for $27.9 \%, 19.56 \%$ and $17.14 \%$. Hong Kong, Macao, Taiwan, and overseas users account for a relatively small proportion.

\subsection{Correlation analysis and regression analysis}

Table 1. Variable correlation table

\begin{tabular}{cccccc}
\hline Variable & Mean & Standard deviation & 1 & 2 & 3 \\
\hline 1. Network size & 91.57 & 122.635 & 1 & 1 & \\
\hline 2. Network density & 1.9673 & 17.4497 & $-0.078^{* *}$ & $-0.059^{*}$ & 1 \\
\hline $\begin{array}{c}\text { 3. Reward sharing } \\
\text { behavior }\end{array}$ & 112.31 & 204.45 & -0.033 & & \\
\hline
\end{tabular}

Note: **. Significant correlation at 0.01 level (bilateral); *. Significant correlation at 0.05 level (bilateral).

From table 1, it can be seen that network density and reward sharing behavior are significantly negatively correlated at 0.05 level, and the correlation coefficient is -0.059 . There is no correlation between network size and reward sharing behavior, and the correlation coefficient is -0.033 .

Table 2. Regression analysis results table

\begin{tabular}{ccc}
\hline & \multicolumn{2}{c}{ Reward sharing behavior } \\
\hline & Model 1 & Model 2 \\
\hline Age & -0.024 & -0.013 \\
\hline Gender & 0.036 & 0.029 \\
\hline Sina V & -0.013 & -0.019 \\
\hline Province & -0.016 & -0.021 \\
\hline Network size & & -0.034 \\
\hline Network density & & $0.055^{*}$ \\
\hline $\mathrm{F}$ & 0.87 & $34.807^{* * *}$ \\
\hline $\mathrm{R}^{2}$ & 0.002 & 0.136 \\
\hline$\triangle \mathrm{F}$ & & $68.61^{* * *}$ \\
\hline$\triangle \mathrm{R}^{2}$ & & 0.134
\end{tabular}

From the regression analysis results, we can see that there is no significant relationship between network size and reward sharing behavior $(\beta=-0.034, \mathrm{p}>0.05)$, hypothesis $\mathrm{H} 1$ has not been verified, that users of large-scale networks in social media may not necessarily share information under the influence of incentives. There is a significant positive correlation between network density and reward sharing behavior $(\beta=0.055, \mathrm{p}<0.05$ ), hypothesis $\mathrm{H} 2$ is confirmed, which indicates that the more connections user has in social media, the easier it is to share information under incentives.

\section{Summary}

This paper, based on the social network theory, puts forward the hypothesis that network size affects reward sharing behavior, but the conclusions do not confirm it. This is consistent with the findings of 
Toubia Olivier, who indicated that users' sharing behavior is influenced by internal utility, and larger audience size will inspire users to post content, but it will also inhibit the enthusiasm. But the conclusion of this article is different from the results of Lingling's research on the sharing behavior, probably because blogger in Sina Micro-blog are more focused on the quality of post, and don't want to disappoint the followers which resulting in a decrease in the number of fans.

The results show that network density in Sina Micro-blog is positively affecting users' reward sharing behavior, which is inconsistent with the previous scholars' research conclusions on WeChat users' sharing behavior [9], the reason may be due to differences in platform, sample, or shared content. In Sina micro-blog, a dense network means more interactions between users and fans, and it has a high degree of intimacy and familiarity, there is a high probability of positive feedback from publishing reward sharing behavior. High density network environment helps reduce face risk, and can also enhance self-expression, gain economic benefits and honorary gains (i.e., knowledgeable, willing to share). Some studies argue that Sina Micro-blog has small world characteristics, and such tight network structure is more conducive for information dissemination [8]. Users in this familiar "small circle" are relatively easy to relax themselves, and will be cautious in unfamiliar situations.

\section{References}

[1] P. Chatterjee, Drivers of new product recommending and referral behavior on social network sites, International Journal of Advertising, vol. 30, pp. 77-101, 2011.

[2] S. E. Arnold, Twitter can lower marketing costs, Smart Business Chicago, 2010.

[3] Y. Sun, Motivation of user-generated content contribution: social connectedness moderates the effects of monetary rewards, Marketing Science, vol. 36, 2016.

[4] S. Erdelez and K. Rioux, Sharing information encountered for others on the web, New Review of Information Behavior Research, vol. 1, 2000.

[5] H. F. Lin, Effects of extrinsic and intrinsic motivation on employee knowledge sharing intentions, Journal of Information Science, vol. 33, pp. 135-149, 2007.

[6] Z. Hui, H. Liaogang, and Y. Zhenpeng, Positive award changes online reviews of consumers? - Review of the impact of reward programs in online word-of-mouth, Management Review, vol. 2, pp. 117-126, 2018.

[7] Y. Danqing, Z. Lu, Blau, and W. Andre, A preliminary analysis of the social network of urban residents in Tianjin - A comparison with the American Social Network, Chinese Social Sciences, vol. 2, pp. 157-176, 1990.

[8] D. Hongtao, M. Qingguo, and W. Junze, Research on social network structure and propagation characteristics of Weibo community based on social network analysis, Journal of the China Society for Scientific and Technical Information, vol. 35, pp. 838-847, 2016.

[9] Y. Lingling, Z. Mi, Z. Xiping, and L. Qian, User content creation and sharing behaviors in mobile social networks: interaction between social networks and narcissism, Journal of the China Society for Scientific and Technical Information, vol. 35, pp. 1000-1008, 2016.

[10]H. Lin, and L. Qiu, Sharing emotion on Facebook: network size, density, and individual motivation, Chi 12 Extended Abstracts on Human Factors in Computing Systems ACM, pp. 2573-2578, 2012.

[11]N. Park, B. Jin, and S.A.A. Jin, Effects of self-disclosure on relational intimacy in Facebook, Computers in Human Behavior, vol. 27, pp. 1974-1983, 2011. 